\title{
Assessment of nicotine dependence among tobacco users visiting outreach programs in Dharan, Nepal: a cross-sectional study
}

Krishna Subedi ( $\square$ idebusanhsirk.ks@gmail.com )

Pokhara Academy of Health Sciences,Pokhara,Nepal https://orcid.org/0000-0001-5409-1751

\section{Ashish Shrestha}

B.P.Koirala Institute of Health Sciences,Dharan,Nepal

\section{Tarakant Bhagat}

B.P.Koirala Institute of Health Sciences, Dharan, Nepal

\section{Research article}

Keywords: FTND, Nicotine dependence, Smokeless tobacco, Smokers, Tobacco prevalence

Posted Date: June 17th, 2020

DOI: https://doi.org/10.21203/rs.3.rs-33461/v1

License: (c) (i) This work is licensed under a Creative Commons Attribution 4.0 International License.

Read Full License

Version of Record: A version of this preprint was published at BMC Public Health on August 6th, 2021. See the published version at https://doi.org/10.1186/s12889-021-11535-9. 


\section{Abstract \\ Background}

Nicotine is a highly addictive substance present in tobacco. This study was conducted to assess the prevalence of tobacco use and compare the nicotine dependence among smokers and smokeless tobacco users visiting dental outreach programs of B.P. Koirala Institute of Health Sciences -Dharan, Nepal.

\section{Methods}

A cross sectional study was conducted from June 2018 to April 2019. A total of 726 people were selected from participants of dental outreach programs of 6 districts using convenience sampling technique. The data collection was done using semi-structured questionnaire through face to face interview by a single researcher. History of tobacco use and level of nicotine dependency was measured using Nepali translated and validated form of Fagerström Test for Nicotine Dependence for smoking and smokeless. The mean age of the tobacco users was $39.55 \pm 15.57$ (Range $=19-82$ years). Descriptive statistics including the mean, median, percentage, standard deviations and interquartile range were computed. Chisquare test, Fisher's exact test and odds ratio were calculated where needed.

\section{Results}

The overall prevalence of any tobacco use, smoking form, smokeless and both form was found to be $32.8 \%, 14.9 \%, 14.3 \%$ and $3.6 \%$ respectively. Nicotine dependence (moderate and severe) was found in $80 \%$ of smokeless tobacco users and $48 \%$ of smokers. Among the smokeless tobacco users nicotine dependency was found to be more with female gender (Odds ratio $=4.98,95 \% \mathrm{Cl}=1.41-17.69, \mathrm{p}=0.01$ ), increase in duration of tobacco use with every 10 years, low socioeconomic status (Odds ratio $=2.75$, $95 \% \mathrm{Cl}=1.02-7.40, \mathrm{p}=0.04$ ), married, not using alcohol. Among smokers nicotine dependency was found to be significantly higher with more than 30 years of duration (Odds ratio $=3.35,95 \% \mathrm{Cl}=1.42-7.92, p=$ 0.005).

\section{Conclusions}

The study concluded that prevalence of tobacco consumption and nicotine dependence was high among the people visiting dental outreach programs. This study incorporated both smoking and smokeless tobacco users. It is high time to develop a policy to control tobacco use along with creating tobacco cessation centers. Tobacco control program are basically focusing on smoking. However, it is very necessary to incorporate in policy level to control smokeless tobacco along with smoking. Tobacco cessation centers will obviously help to quit tobacco among the tobacco users. 


\section{Background}

Tobacco use causes premature death and disease worldwide which is mostly preventable [1]. The world has faced tobacco as an epidemic which is considered as one of the major public health threats to the entire human beings killing more than 8 million people in a year. Most of these deaths are the direct result of tobacco use, consisting of more than 7 million and second-hand smoke consists of around 1.2 million people. There are more than 1 billion smokers residing worldwide, out of which around $80 \%$ are living in middle-and low-income countries (LICs) like India, Bangladesh and Nepal. Tobacco related illness and death is heaviest in these regions as compared to other region of the globe [2]. More than 21,000 children (10-14 years old) and 30,46,000 adults ( $15+$ years old) continue to use tobacco daily in Nepal. More than 27,100 people die each year in Nepal due to tobacco related disease [3].Tobacco use is considered as a severe public health threat in the South-East Asia (SEA) Region. The region has almost one quarter of all smokers in the world [4].

International Classification of Disease (ICD-10) considered tobacco dependence itself as a chronic disease [5].Nicotine is a highly addictive substance, which is present in tobacco along with other 4000 chemicals ${ }^{2}$. Tobacco products in constant use leads to addiction among high proportion of users [6]. Nicotine causes addiction by acting on nicotinic cholinergic receptors which triggers the release of neurotransmitters that produce psychoactive effects that are rewarding [7]. One person prematurely died in every six seconds due to addiction of tobacco. One in two long-term smokers mostly in low- and middle-income countries will die from tobacco addiction. This epidemic reflects the highly addictive nature of tobacco, and specifically of nicotine [8].

There is a decreasing trend of nicotine use in most of the high income countries whereas increasing trend in most of the low and lower-middle income countries [9]. Nicotine dependence is regarded as a primary problem, which is worthy of ongoing attention from every clinician. Complications of nicotine dependence consist of diseases caused by tobacco use or condition worsens by it [6]. Addiction of tobacco is found to be parallel as of heroin and cocaine [10]. Quitting tobacco is not easy, as tobacco dependence is a cluster of physical, behavioral, and psychological or emotional phenomena [11].A cross sectional study done in Nepal showed that nicotine dependence among smokers was found to be $20.4 \%$ and $30.3 \%$ as high and moderate respectively [12]. Various studies have been done related to tobacco in Nepal but there is dearth of studies regarding nicotine dependence among tobacco users.

Tobacco cessation intervention strategies have not been implemented effectively in hospitals and community in Nepal. Till now, there is only one tobacco cessation clinic in Nepal which provides only counselling but not pharmacotherapy. It is of great importance to assess the nicotine dependence among smokers and smokeless tobacco (ST) users which would also help in an attempt in creating a new avenue for the tobacco cessation center in College of Dental surgery (CODS), B.P. Koirala Institute of Health Sciences (BPKIHS) as well as in the entire nation. Therefore this study was done to assess the prevalence of tobacco use and compare nicotine dependence among smokers and smokeless tobacco users visiting outreach programs of the Department of Public Health Dentistry, BPKIHS-Dharan, Nepal. 


\section{Methods}

\section{Study design and duration of study}

A questionnaire based cross sectional study was conducted from June 2018 to April 2019 among people age more than 18 years who visited outreach programs of the Department of Public Health Dentistry, CODS, BPKIHS - Dharan, Nepal including dental camps. BPKIHS is an autonomous Health Sciences University which is located at eastern part of Nepal in province one. The shortest distance from capital city (Kathmandu) of Nepal to Dharan through road is $379 \mathrm{~km}$ which takes around 8 hour and 35 minutes. It is the only Government University with tertiary care in eastern part of Nepal. It has extended its continued health services through teaching district concept to primary health care centers, district hospitals and zonal Hospitals in different parts of the region. It has four colleges: Medical, Dental, Nursing, and Public Health with a 700-bedded Central Teaching Hospital. Study was conducted during regular outreach programs of Department of Public Health Dentistry including dental camps.

\section{Study sites and sampling:}

In this study, data collection was done in five districts out of 14 districts in state number one and one district from state number two using convenience sampling technique. Outreach programs of the department of public health dentistry included two primary health care center, one family health care center, two health post and the dental camps in 13 various sites. Out of these centers three were located in urban areas, two were located in rural areas and all the dental camps were organized in rural areas.

\section{Eligibility Criteria:}

People aged more than 18 years who had visited outreach programs of the Department of Public Health Dentistry, BPKIHS-Dharan, Nepal including dental camps were included in this study. Severe medical conditions affecting their physical or mental health were excluded.

\section{Ethical approval:}

Ethical approval was obtained from the Institutional Review Committee, B.P.Koirala Institute of Health Sciences, Dharan, Nepal (Ref. No: 398/074/075-IRC and Code No: IRC/1191/017)..Signed informed consent was obtained from each participant.

\section{Sample size:}

The sample size was calculated by taking the prevalence of tobacco use in Nepal (30.8\%) [13]; considering $95 \% \mathrm{Cl}, 5 \%$ absolute precision and $15 \%$ non-response rate calculated sample size was 378 . As convenience method of sampling was used, the number from each center was not predetermined. Sample collection was done till the sample size was reached.

\section{Demographic Characteristics}


Demographic variables were age, sex, occupation, marital status, education (illiterate, primary level, middle class level, secondary level, high school level and graduate or postgraduate) income of the family and socioeconomic status (SES) was calculated using Kuppuswamy and classified as per the modifications done in the year 2009 (Ghosh and Ghosh, 2009) using current consumer price index for the year 2017. The current consumer price index was obtained online from Nepal Rastra Bank website (Nepal RB 2017) and the conversion factor was calculated (Conversion factor = Consumer Price Index 2017 divided by Consumer Price Index of 1976). The computed conversion factor was 26.7 (114.8/4.3). For simplicity, SES was categorized into upper (26-29), middle (11-25) and lower $(\leq 5-10)$ class.

The following forms of tobacco use were recorded for tobacco prevalence. Smoking: manufactured cigarettes, cigar, bidis and pipe. Tobacco chewing: khaini, gutka, loose-leaf of tobacco, and gul.

\section{Nicotine dependence}

A standard questionnaire proforma of Fagerstrom Test for Nicotine Dependence (FTND Revised Version) for smoking given by Heatherton et al.(1991) and smokeless form of tobacco given by Ebbert et al. (2006) was translated and validated in Nepali language through standard WHO guidelines. During back translation, the meanings of some questions were modified, so the questions were modified keeping the same scoring criteria (Appendix 1). This modified standard translated and validated questionnaire in Nepali language was administered to each participant by face-to-face interview by the single investigator. Each Smoking and smokeless tobacco questionnaire consisted of 6 questions, carrying score/point based on the answer given. The score ranged from 0 to 10 . Based on the calculated score dependency was categorized as highly dependent (7-10), moderately dependent (4-6) and minimally dependent (<4). Further it was categorized into nicotine dependency (highly and moderately dependent) and no nicotine dependency (minimally dependent).

The higher the Fagerström score, the more intense was the patient's physical dependence on nicotine. Higher scores indicated that treatment of withdrawal symptoms, usually with nicotine replacement therapy, will be an important factor in the patient's plan of care.

Operational definitions: a. Current tobacco user: Used tobacco frequently/ few last days within 1 month prior to examination. b. Nontobacco users: Never used tobacco or occasionally use tobacco. c. Extobacco user: Stopped more than 1 month prior to the examination. d. Smoking behavior. It consisted of information about the number of cigarette/bidis smoked per day, age at smoking initiation, duration of the smoking, family history of tobacco use, ever tried to quit smoking (yes/no) and the number of times tried to quit in last one year. e. Tobacco chewing behavior: It consisted of information about packets of smokeless tobacco used per day, age at tobacco chewing initiation, duration of tobacco chewing, family history of tobacco use, ever tried to quit chewing (yes/no) and the number of times tried to quit in last one year.

All the subjects were advised for smoking/tobacco cessation and tobacco cessation counselling was given to every one after administration of questionnaire. Behavioral cognitive therapy was given involving 
5 " $\mathrm{A}$ " and 5 "R" technique.

\section{Statistical Analysis:}

After completion of the survey, data obtained were entered in Microsoft Excel Sheet version 2007 and analyzed using the Statistical Package for Social Sciences (SPSS version 11.5). Descriptive statistics including the mean, median, percentage, standard deviations and interquartile range were computed. FTND score were categorized as high, moderate and minimal dependent. Further it was categorized as nicotine dependent (high and moderate) and nicotine not dependent (minimal dependent) for analysis of association. Chi-square test, Fisher's exact test and odds ratio were calculated where needed. Odds ratio were calculated using Medcalc's odds ratio calculator. 
Table 1

Socio-demographic characteristics of the tobacco users visiting outreach program of Dharan, Nepal in June 2018 to April $2019(\mathrm{n}=238)$

\begin{tabular}{|c|c|c|c|c|}
\hline $\begin{array}{l}\text { Socio-demographic and } \\
\text { general profile }\end{array}$ & $\begin{array}{l}\text { Male } n=174 \\
(73.11 \%)\end{array}$ & $\begin{array}{l}\text { Female } n=64 \\
(26.89 \%)\end{array}$ & $\begin{array}{l}\text { Total } n=238 \\
(100.0 \%)\end{array}$ & $P$ value \\
\hline Age in years & $77(44.3 \%)$ & $5(7.8 \%)$ & $82(34.5 \%)$ & $x^{2}=96.76$ \\
\hline$\leq 29$ & $36(20.7 \%)$ & $10(15.6 \%)$ & $46(19.3 \%)$ & $P=0.0001$ \\
\hline $30-39$ & $22(12.6 \%)$ & $17(26.6 \%)$ & $39(16.4 \%)$ & \\
\hline $40-49$ & $23(13.2 \%)$ & $20(31.3 \%)$ & $43(18.1 \%)$ & \\
\hline $50-59$ & $16(9.2 \%)$ & $12(18.8 \%)$ & $28(11.8 \%)$ & \\
\hline \multicolumn{5}{|l|}{60} \\
\hline Mean age $\pm S D$ & $36.12 \pm 15.40$ & $48.89 \pm 11.84$ & $39.55 \pm 15.57$ & \\
\hline Marital Status & $57(32.8 \%)$ & $0(0.0 \%)$ & $57(24.0 \%)$ & \multirow{2}{*}{$\begin{array}{l}\text { Fisher exact } \\
\text { value }=27.45 \\
P=0.000\end{array}$} \\
\hline $\begin{array}{l}\text { Unmarried } \\
\text { Married }\end{array}$ & $117(67.2 \%)$ & $64(100.0 \%)$ & $181(76.0 \%)$ & \\
\hline Levels of Education & $12(6.9 \%)$ & $30(46.9 \%)$ & $42(17.6 \%)$ & \multirow{7}{*}{$\begin{array}{l}\text { Fisher exact } \\
\text { value }=87.92 \\
P=0.000\end{array}$} \\
\hline Illiterate & $19(10.9 \%)$ & $16(25.0 \%)$ & $35(14.7 \%)$ & \\
\hline Primary Level( $\leq$ class 5 ) & $21(12.1 \%)$ & $13(20.3 \%)$ & $34(14.3 \%)$ & \\
\hline Middle (6-8) & $42(24.1 \%)$ & $2(3.1 \%)$ & $44(18.5 \%)$ & \\
\hline Secondary Level (SLC) & $66(37.9 \%)$ & $2(3.1 \%)$ & $68(28.6 \%)$ & \\
\hline $\begin{array}{l}\text { Higher secondary Level } \\
(+2 / I A)\end{array}$ & $14(8.0 \%)$ & $1(1.6 \%)$ & $15(6.3 \%)$ & \\
\hline \multicolumn{4}{|l|}{ Graduate/Postgraduate } & \\
\hline \multicolumn{5}{|l|}{ Occupation } \\
\hline Employed & $22(12.6 \%)$ & $2(3.1 \%)$ & $24(10.1 \%)$ & \multirow{6}{*}{$\begin{array}{l}\text { Fisher exact } \\
\text { value }=21.61 \\
P=0.000\end{array}$} \\
\hline Agriculture/Shop & $63(36.2 \%)$ & $36(56.3 \%)$ & $99(41.6 \%)$ & \\
\hline Skilled Worker & $21(12.1 \%)$ & 0 & $21(8.8 \%)$ & \\
\hline Unskilled Worker & $3(1.7 \%)$ & 0 & $3(1.3 \%)$ & \\
\hline Economically inactive & $55(31.6 \%)$ & $25(39.1 \%)$ & $80(33.6 \%)$ & \\
\hline Unemployed & $10(5.7 \%)$ & $1(1.6 \%)$ & $11(4.6 \%)$ & \\
\hline
\end{tabular}




\begin{tabular}{|lllll|}
\hline $\begin{array}{l}\text { Socio-demographic and } \\
\text { general profile }\end{array}$ & $\begin{array}{l}\text { Male } n=174 \\
(73.11 \%)\end{array}$ & $\begin{array}{l}\text { Female } n=64 \\
(\mathbf{2 6 . 8 9 \% )}\end{array}$ & $\begin{array}{l}\text { Total } n=238 \\
(100.0 \%)\end{array}$ & P value \\
\hline Socio-Economic class & $41(23.6 \%)$ & $44(68.8 \%)$ & $85(35.7 \%)$ & $\chi 2=41.61$ \\
Lower & $133(76.4 \%)$ & $20(31.3 \%)$ & $153(64.3 \%)$ & $\mathrm{P}<0.001$ \\
Middle & & & & \\
\hline
\end{tabular}


Table 2

Percentage of males and females who responded to FTND categories (Smoking form) visiting outreach program of Dharan, Nepal in June 2018 to April $2019(n=134)$

\begin{tabular}{|c|c|c|c|c|c|}
\hline Questions & Answers & $\begin{array}{l}\text { Male } \\
(n= \\
107)\end{array}$ & $\begin{array}{l}\text { Female } \\
(n= \\
27)\end{array}$ & $\begin{array}{l}\text { Total } \\
(n= \\
134)\end{array}$ & $\begin{array}{l}P \\
\text { value }\end{array}$ \\
\hline \multirow[t]{4}{*}{$\begin{array}{l}\text { 1.when do you smoke your first cigarette } \\
\text { after waking up? }\end{array}$} & $\begin{array}{l}\text { Within } 5 \\
\text { minutes }\end{array}$ & $\begin{array}{l}29 \\
(27.1 \%)\end{array}$ & $\begin{array}{l}11 \\
(40.7 \%)\end{array}$ & $\begin{array}{l}40 \\
(29.9 \%)\end{array}$ & \multirow{2}{*}{$\begin{array}{l}\text { Fisher } \\
\text { exact } \\
\text { value } \\
=2.80\end{array}$} \\
\hline & $\begin{array}{l}6-30 \\
\text { minutes }\end{array}$ & $\begin{array}{l}20 \\
(18.7 \%)\end{array}$ & $\begin{array}{l}6 \\
(22.2 \%)\end{array}$ & $\begin{array}{l}26 \\
(19.4 \%)\end{array}$ & \\
\hline & $\begin{array}{l}31-60 \\
\text { minutes }\end{array}$ & $\begin{array}{l}20 \\
(18.7 \%)\end{array}$ & $\begin{array}{l}4 \\
(14.8 \%)\end{array}$ & $\begin{array}{l}24 \\
(17.9 \%)\end{array}$ & \multirow{2}{*}{$\begin{array}{l}P= \\
0.42\end{array}$} \\
\hline & $\begin{array}{l}\text { After } 60 \\
\text { minutes }\end{array}$ & $\begin{array}{l}38 \\
(35.5 \%)\end{array}$ & $\begin{array}{l}6 \\
(22.2 \%)\end{array}$ & $\begin{array}{l}44 \\
(32.8 \%)\end{array}$ & \\
\hline \multirow{2}{*}{$\begin{array}{l}\text { 2.Do you feel difficult when you are not } \\
\text { allowed to smoke in the smoking restricted } \\
\text { areas (like temple, library, Cinema hall etc.) }\end{array}$} & Yes & $\begin{array}{l}32 \\
(29.9 \%)\end{array}$ & $\begin{array}{l}9 \\
(33.3 \%)\end{array}$ & $\begin{array}{l}41 \\
(30.6 \%)\end{array}$ & \multirow{2}{*}{$\begin{array}{l}X 2= \\
0.11 \\
P= \\
0.73\end{array}$} \\
\hline & No & $\begin{array}{l}75 \\
(70.1 \%)\end{array}$ & $\begin{array}{l}18 \\
(66.7 \%)\end{array}$ & $\begin{array}{l}93 \\
(69.4 \%)\end{array}$ & \\
\hline \multirow[t]{2}{*}{$\begin{array}{l}\text { 3.Which cigarette is most difficult for you to } \\
\text { quit? }\end{array}$} & $\begin{array}{l}\text { The first } \\
\text { one in the } \\
\text { morning }\end{array}$ & $\begin{array}{l}63 \\
(58.9 \%)\end{array}$ & $\begin{array}{l}18 \\
(66.7 \%)\end{array}$ & $\begin{array}{l}81 \\
(60.4 \%)\end{array}$ & \multirow{2}{*}{$\begin{array}{l}X 2= \\
0.54 \\
P= \\
0.46\end{array}$} \\
\hline & Any other & $\begin{array}{l}44 \\
(41.1 \%)\end{array}$ & $\begin{array}{l}9 \\
(33.3 \%)\end{array}$ & $\begin{array}{l}53 \\
(39.6 \%)\end{array}$ & \\
\hline \multirow[t]{3}{*}{$\begin{array}{l}\text { 4. How many cigarettes do you smoke per } \\
\text { day? }\end{array}$} & 10 or less & $\begin{array}{l}90 \\
(84.1 \%)\end{array}$ & $\begin{array}{l}21 \\
(77.8 \%)\end{array}$ & $\begin{array}{l}111 \\
(82.8 \%)\end{array}$ & \multirow{2}{*}{$\begin{array}{l}\text { Fisher } \\
\text { exact } \\
\text { value } \\
=3.39\end{array}$} \\
\hline & $11-20$ & $\begin{array}{l}17 \\
(15.9 \%)\end{array}$ & $\begin{array}{l}5 \\
(18.5 \%)\end{array}$ & $\begin{array}{l}22 \\
(16.4 \%)\end{array}$ & \\
\hline & $21-30$ & $0(\%)$ & $\begin{array}{l}1 \\
(3.7 \%)\end{array}$ & $\begin{array}{l}1 \\
(0.7 \%)\end{array}$ & 0.21 \\
\hline \multirow{2}{*}{$\begin{array}{l}\text { 5. Do you smoke more frequently during the } \\
\text { first hours after waking than during the rest } \\
\text { of the day? }\end{array}$} & Yes & $\begin{array}{l}11 \\
(10.3 \%)\end{array}$ & $\begin{array}{l}6 \\
(22.2 \%)\end{array}$ & $\begin{array}{l}17 \\
(12.7 \%)\end{array}$ & $\begin{array}{l}\chi 2= \\
2.77\end{array}$ \\
\hline & No & $\begin{array}{l}96 \\
(89.7 \%)\end{array}$ & $\begin{array}{l}21 \\
(77.8 \%)\end{array}$ & $\begin{array}{l}117 \\
(87.3 \%)\end{array}$ & $\begin{array}{l}P= \\
0.09\end{array}$ \\
\hline \multirow[t]{2}{*}{$\begin{array}{l}\text { 6. Do you smoke even if you are ill and } \\
\text { bedridden most of the day? }\end{array}$} & Yes & $\begin{array}{l}56 \\
(52.3 \%)\end{array}$ & $\begin{array}{l}20 \\
(74.1 \%)\end{array}$ & $\begin{array}{l}76 \\
(56.7 \%)\end{array}$ & $\begin{array}{l}x 2= \\
4.15\end{array}$ \\
\hline & No & $\begin{array}{l}51 \\
(47.7 \%)\end{array}$ & $\begin{array}{l}7 \\
(25.9 \%)\end{array}$ & $\begin{array}{l}58 \\
(43.3 \%)\end{array}$ & $\begin{array}{l}P= \\
0.04\end{array}$ \\
\hline
\end{tabular}


Table 3

Percentage of males and females who responded to FTND categories (Smokeless form) visiting outreach program of Dharan, Nepal in June 2018 to April $2019(n=130)$

\begin{tabular}{|c|c|c|c|c|c|}
\hline Questions & Answers & $\begin{array}{l}\text { Male } \\
(n= \\
86)\end{array}$ & $\begin{array}{l}\text { Female } \\
(n=44)\end{array}$ & $\begin{array}{l}\text { Total } \\
(n=130)\end{array}$ & $\begin{array}{l}P \\
\text { value }\end{array}$ \\
\hline \multirow[t]{4}{*}{$\begin{array}{l}\text { 1. when do you chew the first tobacco } \\
\text { after you wake up? }\end{array}$} & $\begin{array}{l}\text { Within } 5 \\
\text { minutes }\end{array}$ & $\begin{array}{l}22 \\
(25.6 \%\end{array}$ & $\begin{array}{l}16 \\
(36.4 \%)\end{array}$ & $\begin{array}{l}38 \\
(29.2 \%)\end{array}$ & \multirow{4}{*}{$\begin{array}{l}X 2= \\
4.52 \\
P= \\
0.21\end{array}$} \\
\hline & $\begin{array}{l}6-30 \\
\text { minutes }\end{array}$ & $\begin{array}{l}24 \\
(27.9 \%\end{array}$ & $\begin{array}{l}13 \\
(29.5 \%\end{array}$ & $\begin{array}{l}37 \\
(28.5 \%)\end{array}$ & \\
\hline & $\begin{array}{l}31-60 \\
\text { minutes }\end{array}$ & $\begin{array}{l}12 \\
(14.0 \%\end{array}$ & )$^{8}(18.2 \%$ & $20(15.4 \%$ & \\
\hline & $\begin{array}{l}\text { After } 60 \\
\text { minutes }\end{array}$ & $\begin{array}{l}28 \\
(32.6 \%\end{array}$ & )$^{7}(15.9 \%$ & $\begin{array}{l}35 \\
(26.9 \%)\end{array}$ & \\
\hline \multirow[t]{3}{*}{$\begin{array}{l}\text { 2. How often do you intentionally swallow } \\
\text { tobacco juice? }\end{array}$} & Always & $\begin{array}{l}12 \\
(14.0 \%\end{array}$ & $\begin{array}{l}5 \\
(11.4 \%\end{array}$ & $\begin{array}{l}17 \\
(13.1 \%)\end{array}$ & \multirow{3}{*}{$\begin{array}{l}\chi 2= \\
0.38 \\
P= \\
0.82\end{array}$} \\
\hline & Sometimes & $\begin{array}{l}52 \\
(60.5 \%\end{array}$ & $\begin{array}{l}29 \\
(65.9 \%\end{array}$ & $\begin{array}{l}81 \\
(62.3 \%)\end{array}$ & \\
\hline & Never & $\begin{array}{l}22 \\
(25.6 \%\end{array}$ & $\begin{array}{l}10 \\
(22.7 \%\end{array}$ & $\begin{array}{l}32 \\
(24.6 \%)\end{array}$ & \\
\hline \multirow[t]{2}{*}{$\begin{array}{l}\text { 3. which tobacco is most difficult for you } \\
\text { to quit? }\end{array}$} & $\begin{array}{l}\text { The first one } \\
\text { in the } \\
\text { morning }\end{array}$ & $\begin{array}{l}40 \\
(46.5 \%\end{array}$ & $\begin{array}{l}34 \\
(77.3 \%\end{array}$ & $\begin{array}{l}74 \\
(56.9 \%)\end{array}$ & \multirow{2}{*}{$\begin{array}{l}X 2= \\
11.23 \\
P= \\
0.001\end{array}$} \\
\hline & Any other & $\begin{array}{l}46 \\
(53.5 \%\end{array}$ & $\begin{array}{l}10 \\
(22.7 \%\end{array}$ & $\begin{array}{l}56 \\
(43.1 \%)\end{array}$ & \\
\hline \multirow[t]{3}{*}{$\begin{array}{l}\text { 4. How many packets/cans of tobacco do } \\
\text { you use per week? }\end{array}$} & More than 3 & $\begin{array}{l}52 \\
(60.5 \%\end{array}$ & $\begin{array}{l}19 \\
(43.2 \%\end{array}$ & $\begin{array}{l}71 \\
(54.6 \%)\end{array}$ & \multirow{3}{*}{$\begin{array}{l}X 2= \\
4.04 \\
P= \\
0.13\end{array}$} \\
\hline & $2-3$ & $\begin{array}{l}23 \\
(26.7 \%\end{array}$ & $\begin{array}{l}19 \\
(43.2 \%\end{array}$ & $\begin{array}{l}42 \\
(32.3 \%)\end{array}$ & \\
\hline & 1 & $\begin{array}{l}11 \\
)^{112.8 \%}\end{array}$ & )$^{6}(13.6 \%$ & $\begin{array}{l}17 \\
(13.1 \%)\end{array}$ & \\
\hline
\end{tabular}




\begin{tabular}{|c|c|c|c|c|c|}
\hline Questions & Answers & $\begin{array}{l}\text { Male } \\
(n= \\
86)\end{array}$ & $\begin{array}{l}\text { Female } \\
(n=44)\end{array}$ & $\begin{array}{l}\text { Total } \\
(n=130)\end{array}$ & $\begin{array}{l}P \\
\text { value }\end{array}$ \\
\hline \multirow[t]{2}{*}{$\begin{array}{l}\text { 5. Do you chew tobacco more in the first } \\
\text { one hour after you wake up than during } \\
\text { rest of the day? }\end{array}$} & Yes & )$^{15}(17.4 \%$ & )$^{7}(15.9 \%$ & $\begin{array}{l}22 \\
(16.9 \%)\end{array}$ & $\begin{array}{l}x 2= \\
0.04\end{array}$ \\
\hline & No & $\begin{array}{l}71 \\
(82.6 \%\end{array}$ & $\begin{array}{l}37 \\
(84.1 \%\end{array}$ & $\begin{array}{l}108 \\
(83.1 \%)\end{array}$ & $\begin{array}{l}P= \\
0.82\end{array}$ \\
\hline \multirow[t]{2}{*}{$\begin{array}{l}\text { 6. Do you chew tobacco even if you are ill } \\
\text { and bedridden most of the day? }\end{array}$} & Yes & $\begin{array}{l}61 \\
(70.9 \%\end{array}$ & $\begin{array}{l}39 \\
(88.6 \%\end{array}$ & $\begin{array}{l}100 \\
(76.9 \%)\end{array}$ & \multirow{2}{*}{$\begin{array}{l}X 2= \\
5.14 \\
P= \\
0.02\end{array}$} \\
\hline & No & $\begin{array}{l}25 \\
(29.1 \%\end{array}$ & $\begin{array}{l}5 \\
(11.4 \%\end{array}$ & $\begin{array}{l}30 \\
(23.1 \%)\end{array}$ & \\
\hline
\end{tabular}

Table 4

Classification of FTND score for smoking and smokeless tobacco (ST) users visiting outreach program of Dharan, Nepal in June 2018 to April $2019(n=238)$

\begin{tabular}{|c|c|c|c|c|c|c|}
\hline \multirow{2}{*}{$\begin{array}{l}\text { FTND } \\
\text { Classification }\end{array}$} & \multicolumn{2}{|l|}{ Male $(n=107)$} & \multicolumn{2}{|c|}{ Female $(n=27)$} & \multicolumn{2}{|l|}{ Total $(n=134)$} \\
\hline & $\begin{array}{l}\text { Smoking }(\mathrm{n}= \\
107)\end{array}$ & $\begin{array}{l}S T \\
86)\end{array}(n=$ & $\begin{array}{l}\text { Smoking ( } \mathrm{n} \\
=27 \text { ) }\end{array}$ & $\begin{array}{l}\text { ST }(n= \\
44)\end{array}$ & $\begin{array}{l}\text { Smoking }(n= \\
134)\end{array}$ & $\begin{array}{l}\text { ST }(n= \\
130)\end{array}$ \\
\hline Minimal $(<4)$ & $59(55.1 \%)$ & $\begin{array}{l}23 \\
(26.7 \%)\end{array}$ & $11(40.7 \%)$ & $\begin{array}{l}3 \\
(6.8 \%)\end{array}$ & $70(52.2 \%)$ & $\begin{array}{l}26 \\
(20.0 \%)\end{array}$ \\
\hline Medium (4-6) & $39(36.4 \%)$ & $\begin{array}{l}36 \\
(41.9 \%)\end{array}$ & $13(48.1 \%)$ & $\begin{array}{l}24 \\
(54.5 \%)\end{array}$ & $52(38.8 \%)$ & $\begin{array}{l}60 \\
(46.2 \%)\end{array}$ \\
\hline High $(7-10)$ & $9(8.4 \%)$ & $\begin{array}{l}27 \\
(31.4 \%)\end{array}$ & $3(11.1 \%)$ & $\begin{array}{l}17 \\
(38.6 \%)\end{array}$ & $12(9.0 \%)$ & $\begin{array}{l}44 \\
(33.8 \%)\end{array}$ \\
\hline
\end{tabular}


Table 5

Classification of FTND score for smoking and smokeless tobacco (ST) users visiting outreach program of Dharan, Nepal in June 2018 to April $2019(n=238)$ based on background characteristics

\begin{tabular}{|c|c|c|c|c|c|c|c|}
\hline \multicolumn{5}{|c|}{ Smoking FTND } & \multicolumn{3}{|c|}{ Smokeless Tobacco FTND } \\
\hline $\begin{array}{l}\text { Independent } \\
\text { correlate (n) }\end{array}$ & $\begin{array}{l}\text { Odds } \\
\text { ratio }\end{array}$ & $\begin{array}{l}95 \% \\
\mathrm{Cl}\end{array}$ & $\begin{array}{l}P \\
\text { value }\end{array}$ & $\begin{array}{l}\text { Independent } \\
\text { correlate (n) }\end{array}$ & $\begin{array}{l}\text { Odds } \\
\text { ratio }\end{array}$ & $95 \% \mathrm{Cl}$ & $\begin{array}{l}\mathrm{P} \\
\text { value }\end{array}$ \\
\hline Gender & & & & Gender & & & \\
\hline Male (107) & Ref & - & - & Male (86) & Ref & - & - \\
\hline Female (27) & 1.78 & $\begin{array}{l}0.76- \\
4.21\end{array}$ & 0.18 & Female (44) & 4.98 & $\begin{array}{l}1.41- \\
17.69\end{array}$ & 0.01 \\
\hline \multicolumn{4}{|c|}{ Mean age of initiation of tobacco use } & \multicolumn{4}{|c|}{ Mean age of initiation of tobacco use } \\
\hline$\leq 20(92)$ & Ref & - & - & $\leq 20(78)$ & Ref & - & - \\
\hline$>20(42)$ & 0.88 & $\begin{array}{l}0.42- \\
1.82\end{array}$ & 0.73 & $>20(52)$ & 1.13 & $\begin{array}{l}0.47- \\
2.69\end{array}$ & 0.78 \\
\hline \multicolumn{4}{|l|}{ Total duration of use } & \multicolumn{4}{|c|}{ Total duration of use } \\
\hline$<10(66)$ & Ref & - & - & $<10(47)$ & Ref & - & - \\
\hline $10-20(18)$ & 1.75 & $\begin{array}{l}0.61- \\
5.00\end{array}$ & 0.29 & $10-20(32)$ & 5.18 & $\begin{array}{l}1.57- \\
17.16\end{array}$ & 0.01 \\
\hline $21-30(15)$ & 2.00 & $\begin{array}{l}0.64- \\
6.20\end{array}$ & 0.22 & $21-30(17)$ & 26.09 & $\begin{array}{l}1.48- \\
459.59\end{array}$ & 0.03 \\
\hline > 30 (35) & 3.35 & $\begin{array}{l}1.42- \\
7.92\end{array}$ & 0.005 & > 30 (34) & 11.85 & $\begin{array}{l}2.54- \\
55.35\end{array}$ & 0.002 \\
\hline \multicolumn{4}{|l|}{ Socioeconomic status } & \multicolumn{4}{|c|}{ Socioeconomic status } \\
\hline Medium (92) & Ref & - & - & Medium (77) & Ref & - & - \\
\hline Low (42) & 1.99 & $\begin{array}{l}0.95- \\
4.19\end{array}$ & 0.06 & Low (53) & 2.75 & $\begin{array}{l}1.02- \\
7.40\end{array}$ & 0.04 \\
\hline Marital Status & & & & Marital Status & & & \\
\hline Married (85) & Ref & - & - & Married (109) & Ref & - & - \\
\hline $\begin{array}{l}\text { Unmarried/single/ } \\
\text { divorced (49) }\end{array}$ & 0.83 & $\begin{array}{l}0.34- \\
2.07\end{array}$ & 0.70 & $\begin{array}{l}\text { Unmarried/ } \\
\text { single/divorced } \\
(21)\end{array}$ & 0.14 & $\begin{array}{l}0.05- \\
0.40\end{array}$ & 0.0002 \\
\hline Occupation & & & & Occupation & & & \\
\hline $\begin{array}{l}\text { Employed-Skilled } \\
\text { (35) }\end{array}$ & Ref & - & - & $\begin{array}{l}\text { Employed- } \\
\text { Skilled (32) }\end{array}$ & Ref & - & - \\
\hline
\end{tabular}




\begin{tabular}{|lcllllll|}
\hline \multicolumn{3}{|c|}{ Smoking FTND } & & \multicolumn{3}{c|}{ Smokeless Tobacco FTND } \\
\hline $\begin{array}{l}\text { Employed-Unskilled } \\
\text { (42) }\end{array}$ & 0.52 & $\begin{array}{l}0.20- \\
1.31\end{array}$ & 0.16 & $\begin{array}{l}\text { Employed- } \\
\text { Unskilled (51) }\end{array}$ & 2.50 & $\begin{array}{l}0.77- \\
8.04\end{array}$ & 0.12 \\
\hline Unemployed (57) & 0.28 & $\begin{array}{l}0.11- \\
0.68\end{array}$ & 0.005 & $\begin{array}{l}\text { Unemployed } \\
(47)\end{array}$ & 0.97 & $0.34-2.73$ & 0.95 \\
\hline Alcohol use & & & & Alcohol use & & & \\
\hline No (49) & Ref & - & - & No (71) & Ref & - & - \\
\hline Yes(85) & 1.36 & $0.67-$ & 0.39 & Yes (59) & 0.31 & $0.13-$ & 0.01 \\
\hline
\end{tabular}

\section{Results}

Out of 726 (406 male and 320 female) people surveyed in the study $238(32.8 \%)$ were tobacco users. The prevalence of tobacco use in any form for male and female was found to be $42.8 \%$ (174) and $20.0 \%$ (64) respectively. The prevalence of smokeless tobacco use was $14.3 \%$ ( $16.5 \%$ for male and $11.6 \%$ for female) and that of smoking was $14.9 \%$ (20.9\% for male versus $6.2 \%$ ). In both form (smoking and smokeless tobacco) tobacco use was prevalent in $3.6 \%$ ( $4.7 \%$ for male and $2.2 \%$ for female). Among the tobacco users, prevalence of tobacco use was found to be higher in males $(73 \% ; 174)$ as compared to females $(27 \% ; 64)$. The age ranged from 18 to 82 years with mean age and standard deviation (S.D) of $39.55 \pm$ 15.57 (male $36.12 \pm 15.40$ and female $48.89 \pm 11.84$ ). More number of married people used tobacco $(74.8 \%)$.The majority of people $(65.1 \%)$ had education of school leaving certificate or lower. Only $6.3 \%$ had graduate/postgraduate level of education. Female had low level of education as compared to male $(p=<0.001)$. The majority of the people belonging to middle socioeconomic class followed by lower socioeconomic class (Table1).

Majority of male were $(88,50.6 \%)$ smokers. Almost $58.0 \%$ female (37) chewed smokeless form of tobacco. The same percentage of male $(19,10.9 \%)$ and female $(7,10.9 \%)$ population consumed both form of tobacco. Majority of the tobacco users $(127,53.4 \%)$ consumed alcohol. Male $(102,58.6 \%)$ consumed more alcohol as compared to female $(25,39.1 \%)$. Alcohol use was seen in more percentage of people who were using both form of tobacco $(73.7 \%$ in male versus $42.8 \%$ in females) followed by smoking ( $64.8 \%$ in male versus $55.0 \%$ in female) and least by ST users ( $46.3 \%$ in male versus $29.7 \%$ in female).

Among the tobacco users $44.5 \%$ (106) had one or more family members who also uses tobacco.

The mean age of initiation of tobacco use for smokeless tobacco was 21.0 \pm 8.8. However, it was earlier in case of smoking with mean age of initiation 19.1 \pm 5.9 . However, female initiated smokeless tobacco earlier than male ( $20.0 \pm 6.8$ vs $21.6 \pm 9.6)$. But male started smoking at earlier age than female $(18.9 \pm 5.2$ Vs $19.9 \pm 8.4)$. 
The median duration of tobacco chewing was 17 years (IQR: 6-31). The median duration of tobacco chewing for male and female was to be 10 years (IQR: 4.7-26) and 25 years (IQR: 14.2-37.7) respectively. The median duration of smoking was 11.5 (IQR: 4-31). The median duration of smoking for male and female was found to be 6 years (IQR: 4-26) and 38 years (IQR: 24-42) respectively.

Only $24.2 \%$ (64) tried to quit tobacco at least once or twice in the last one year. More percentage of male tobacco users $(51,26.4 \%)$ tried to quit in comparison to female $(13,18.3 \%)$.

Among smokers, $29.9 \%(n=40)$ had smoked the first cigarette within 5 minutes whereas $32.8 \%(n=44)$ had smoked after 60 minutes of waking up. Almost $31 \%$ of smokers felt difficult when they were not allowed to smoke in the smoking restricted areas (temple, library, cinema hall etc.). About $60 \%$ smokers felt difficult to quit first cigarette in the morning. Most of the smokers (82.8\%) smoked $\leq 10$ cigarettes per day. Only $12.7 \%$ of smokers smoked more frequently during the first hours after waking up than during the rest of the day. Majority of the female smokers $(74.1 \%, n=20, P=0.04)$ smoked even if they were ill and bedridden most of the day as compared to male (Table 2 ).

Among smokeless tobacco (ST) users, 29.2\% ( $n=38)$ chewed first tobacco within 5 minutes after waking up in the morning. About $13 \%(n=17)$ of ST users intentionally swallowed the tobacco juice. Majority of the female smokeless tobacco users $(77.3 \%, n=34, P=0.001))$ felt difficult to quit first tobacco chewed in the morning as compared to male. More than half of the ST users $(54.6 \%, n=71)$ consumed more than 3 packets/cans of tobacco in a week. Only $17.0 \%$ of the ST users chewed more tobacco in the first one hour after they woke up than during rest of the day. More than $2 / 3^{\text {rd }}$ of ST users chewed tobacco even if they were ill and bedridden most of the day (Table 3).

Nicotine dependence was found in $48 \%$ of smokers. About $52 \%$ had minimal level of nicotine dependence. Nicotine dependence was found to be higher in females (59\%) as compared to males (45\%) (Table 4). Nicotine dependence was found in $80 \%$ of ST users. More than $90 \%$ female had medium or high level of nicotine dependence whereas in male it was found to be $73 \%$ (Table 4).

Nearly $39 \%$ of ST users who initiated tobacco chewing on or before 20 years of age had high level of nicotine dependence as compared to $26.9 \%$ who had initiated after 20 years of age. Smokers who had initiated smoking on or before 20 years of age and after 20 years of age had almost same high level of nicotine dependence (8.7\% vs $9.5 \%)$.

Among the smokeless tobacco users nicotine dependency was found to be more with female gender (Odds ratio $=4.98,95 \% \mathrm{Cl}=1.41-17.69, \mathrm{p}=0.01$ ), increase in duration of tobacco use with every 10 years, low socioeconomic status (Odds ratio $=2.75,95 \% \mathrm{Cl}=1.02-7.40, \mathrm{p}=0.04$ ), married, not using alcohol. Among smokers nicotine dependency was found to be significantly higher with more than 30 years of duration (Odds ratio $=3.35,95 \% \mathrm{Cl}=1.42-7.92, \mathrm{p}=0.005$ ) (Table 5 ).

\section{Discussion}


This study was done to assess the prevalence of tobacco use and level of nicotine dependence in outreach program of BPKIHS-Dharan, Nepal. Tobacco prevalence in this study (32.8\%) was found almost similar to the studies done by STEPS survey [13] in Nepal in 2013 (30.8\%) and Sreeramareddy et al.[14] (2011) (30.3\%) . Tobacco prevalence in male was found to be lower (42.8\%) as compared to the studies done by STEPS survey [13] in Nepal in 2013, Sreeramareddy et al. [14] and NDHS-2016 [15] where it was found to be $48.1 \%, 56.5 \%$, and $52.3 \%$ respectively. It may be due to the fact that various forms of tobacco (betel / betel quid (Paan) and water pipe) were not considered as it was considered in above studies. Tobacco prevalence in current study in female (20\%) was found to be consistent with the studies done by Sreeramareddy et al. [14] (19.6\%). However it was found higher than the studies done by STEPS survey [13]2013 and NDHS-2016 [16] where it was found to be $14.1 \%$ and $8.4 \%$ respectively.

The smoking tobacco pattern in this study was found to be lower as compared to the other studies done by STEPS survey [13] 2013, Sreeramareddy et al. [14] and NDHS-2016 [15] .This may be due to the fact that in this study prevalence of smoking, smokeless tobacco and both form were categorized differently whereas only smoking and smokeless form were categorized in other studies. The prevalence of smoking in male in current study was found to be lower with other studies [13-15] done in Nepal. The prevalence of smoking in female was found to be almost similar to NDHS 2016 [15] (6.2\% vs 5.8\%) whereas it was found to be higher in STEPS survey [13] 2013 (10.3\%).

The prevalence of smokeless tobacco users was found to be similar to Sreeramareddy et al.[14] (14.3\% vs $14.6 \%$ ) whereas it was found to be lower as compared to STEPS survey [13] (17.8\%). The use of smokeless tobacco was found to be low among male and high among female in our study as compared to other studies done by STEPS survey [13] 2013, Sreeramareddy et al. [14] and NDHS-2016 [15].The prevalence of smokeless tobacco users in female was found to be similar to Global Adult Tobacco Survey (GATS): India 2016-17 Report [16] (11.6\% vs 12.8\%). Higher prevalence of smokeless tobacco use in female in this study might be associated with lower level of education. Almost half of the female (47\%) tobacco users in this study were illiterate and very few (8\%) had secondary and higher level of education. The rate of smokeless tobacco was highest among the illiterate and lowest among those with a college education $[17,18]$. Dual (use of both smoking and smokeless tobacco) use of tobacco was almost similar to global Adult Tobacco Survey: India 2016-17 Report [16] (3.6\% vs 3.4\%).

Almost $45 \%$ tobacco users had positive family history of tobacco use. It had been shown that habit of tobacco use run in families [19].

More than half of the tobacco users $(127,53.4 \%)$ also consumed alcohol. Alcohol consumption was found to be more among male $(102,58.6 \%)$ than female $(25,39.1 \%)$. Study also confirmed that co-use of tobacco and alcohol [20]. Mean age of initiation of tobacco smoking was found to be almost similar (19.10 \pm 5.939) to STEPS survey (18.2 years) [13].

About one in three (almost 29\%) tobacco users used the first tobacco immediately or within five minutes of waking up which is in contrast to GATS: India 2016-17 Report [16] where about one in five (18\%) daily 
tobacco users resorted to tobacco use immediately or within five minutes. This indicates that dependency of tobacco was high in our study population.

Almost $48 \%$ had medium and high level of nicotine dependency among smokers which is almost similar to the study done by Aryal et al. [12] where about $51 \%$ had medium and high level of nicotine dependency. However high level of nicotine dependency was found in only $9 \%$ as compared to $20.4 \%$ in Aryal et al [12].This study was conducted exclusively in a community level which comprises individuals from both rural and urban settings.

Till date no any study had been done in Nepal regarding nicotine assessment of smokeless tobacco users. In our study nicotine dependence was seen more in smokeless tobacco users (80\%) as compared to smokers (48\%). Higher median duration of use of ST in comparison to smoking (17years versus 11.5 years) may be one of the factor. Also, it may be due to that smokeless tobacco contains more nicotine as compared to cigarette smoking [21].

Only $24.24 \%$ (64) tried to quit tobacco at least 1 or 2 times in last one year. More percentage of male tobacco users $(51,26.4 \%)$ tried to quit in comparison to female $(13,18.3 \%)$. Evidence suggests that women were significantly less likely to quit smoking than men both due to biological and psychological factors, suggesting that the addictiveness of smoking may be greater for females [22].

The various factors for high level of nicotine dependency include: lower income, lower education, younger age of first smoking [23], high alcohol dependence [23,24], mood and anxiety disorders [25] and genetic factors $[23,26]$. High nicotine dependence is associated with lower quality of life, lower work productivity and higher health-care use [27]. Lower level of education, more duration of tobacco use, history of less previous quit attempt in last one year and elder females may be the factors associated with more nicotine dependency level seen in female in our study. Most of the studies were done considering only smoking form of tobacco whereas our study considered both form of tobacco i.e smoking and ST.

\section{Strengths and Limitations of the study}

First, social desirability bias [28] might have occurred. Social desirability bias refers to the tendency of respondents to give socially desirable responses instead of giving true responses. It becomes a major issue especially in socially sensitive issues such as politics, religion, and environment, or personal issues such as drug use, cheating, and smoking [29]. As the tobacco use status was obtained through self-report without biochemical verification, which might lead to reporting inaccuracy. There may be a possibility of information bias especially related to history of tobacco use and number of cigarettes/tobacco can or pouches used per day. However, Society for Research on Nicotine and Tobacco (SRNT) Subcommittee on Biochemical Verification [30] suggested that in population based studies biochemical verification is neither feasible nor necessary.

Second, the study was based on non-probability sampling (convenience sampling). The effect of outliers can be seen in this kind of subject selection. Outliers are cases whom consider as not belonging to the 
data [31]. Therefore the result shows associations but do not deliver evidence for causality.

Third, this study might hide the true prevalence as smoking by women is socially unacceptable. The cultural and geographical variation might affect tobacco use and level of nicotine dependence [32].

Although the convenience sampling was used, our study covered more than $30 \%$ of the districts of province number one. The data collection was done in various parts of the selected districts. Moreover the results obtained from this study can be generalized in province number one. Further studies are required to confirm the dependency using the bio-markers. A large nationwide study should be done to assess the level of nicotine dependence.

\section{Recommendations}

It is very important to develop a tobacco cessation program. The findings from our study could be a stepping stone for opening of tobacco cessation centers, which can help to reduce the tobacco prevalence as well mortality from oral and other cancer caused due to tobacco use. Health education $(\mathrm{HE})$ can raise the awareness and motivate patients to think about quitting of tobacco use. HE along with nicotine replacement therapy (NRT) should be incorporated to reduce nicotine dependence as well as tobacco use prevalence. In outreach programs health education can impart knowledge about harmful effects of tobacco and raise awareness about quitting to the people in that area. Health education and other protective measures should be taken by government as well as health professionals that discourage tobacco use and help to people to quit tobacco which help to save more number of lives result from deadly consequences of tobacco use.

\section{Conclusions}

Our study concluded that tobacco prevalence was high (32.8\% of the study population). Nicotine dependence (Moderate and severe) was more prevalent in ST users (80\%) in comparison to smokers $(48 \%)$.

\section{Abbreviations}

\section{LICs}

Low-income countries

SEA

South-East Asia

ICD

International Classification of Disease

ST

Smokeless tobacco

\section{CODS}

College of Dental surgery 


\section{BPKIHS}

B.P. Koirala Institute of Health Sciences

SES

Socioeconomic status

FTND

Fagerstrom Test for Nicotine Dependence

SPSS

Statistical Package for Social Sciences

GATS

Global Adult Tobacco Survey

SRNT

Society for Research on Nicotine and Tobacco

NRT

Nicotine replacement therapy

HE

Health Education

\section{Declarations}

\section{Ethics approval and consent to participate}

Ethical approval was obtained from the Institutional Review Committee, B.P.Koirala Institute of Health Sciences, Dharan, Nepal (Ref. No: 398/074/075-IRC and Code No: IRC/1191/017).Signed informed consent was obtained from each participant.

\section{Consent for publication}

Not applicable

\section{Availability of data and materials}

The datasets analyzed during the current study are available from the corresponding author on reasonable request.

Competing interests: The authors declare that they have no competing interest.

Funding: This project had won the grant award worth of Nepalese rupees 25,000 (£165.72) from Nepal Young Health Professionals Training 2017 (No. 201709-05). Nepal Young Health Professionals Training 2017 did not have a role in the planning or interpretation of this study, or in the writing of this manuscript.

\section{Author's contributions}


KS, AS AND TKB substantially contributed by developing conceptual framework, design of the study and involved in drafting, interpretation of data and critically revising the article. KS was involved in data management and analysis of data. All authors read and approved the final version of the manuscript.

\section{Acknowledgements}

This work could not have been successful without the invaluable help from my senior Dr. Santosh Kumari Agrawal (Assistant Professor), colleagues Dr. Abanish Singh, Dr. Ujwal Gautam, Dr. Sudeep Subedi , Dr. Aashma Sapkota, Dr. Naresh Prasad Joshi and Dr. Resham Koirala.

I would like to extend my sincere thanks to all the participants without whom my research work would not have been completed.

\section{References}

1. Centers for disease control and prevention. Global Tobacco Control. https://www.cdc.gov /tobacco/global/index.htm. Accessed June 14,2017.

2. World Health Organization. Tobacco. https://www.who.int/news-room/ fact-sheets/detail/tobacco. Accessed June 4, 2019.

3. The tobacco Atlas. Nepal. https://tobaccoatlas.org /country/nepal/. Accessed June 4, 2019.

4. 10.4103/0019-557X.89943

Thakur JS, Garg R, Narain JP, Menabde N. Tobacco use: a major risk factor for non communicable diseases in South-East Asia region. Indian J Public Health. 2011 Jul 1;55(3):155. doi: 10.4103/0019$557 X .89943$.

5. World Health Organization. The ICD-10 classification of mental and behavioural disorders: clinical descriptions and diagnostic guidelines. Geneva: World Health Organization; 1992.

6. American Society of Addiction Medicine. Public Policy Statement on Nicotine Addiction and Tobacco. https://www.asam.org/ advocacy/find-a-policy-statement/view-policy-statement/publicpolicy-statements/2011/ 12/15/nicotine-addiction-and-tobacco. Accessed June 152017.

7. Benowitz NL. Nicotine addiction. N. Engl. J Med. 2010 Jun;17(24):2295-303. doi:10.1056/NEJMra0809890. 362 ) .

8. World Health Organization. Gender, Women and the Tobacco Epidemic: Addiction to Nicotine. https://www.who.int/tobacco/publications/gender/ en_tfi_gender_women_addiction_nicotine.pdf?ua = 1. Accessed on 18 April 182019.

9. Hospital physician. psychiatry board review manual. Nicotine use disorders. http://www.hospitalphysician.com/pdf/brm_Psy_V8P2.pdf. Accessed May 27, 2017.

10. General S, Wright J, Speaker DH. Nicotine addiction. A report of the Surgeon General. US Dept. of Health and Human Services, Washington DC. 1988. https://profiles.nlm.nih.gov/ps/access/ nnbbzd.pdf. Accessed June 15, 2017. 
11. World Health Organization. A guide for tobacco users to quit. https://apps.who.int/iris/bitstream/handle/10665/112833/9789241506939_eng.pdf?sequence=1. Accessed June 16, 2017.

12. $10.1186 / \mathrm{s} 12971-015-0053-8$

Aryal UR, Bhatta DN, Shrestha N, Gautam A. Assessment of nicotine dependence among smokers in Nepal: a community based cross-sectional study. Tob Induc Dis. 2015 Aug 26;13(1):26. doi:10.1186/s12971-015-0053-8.

13. Aryal KK. Non communicable diseases risk factors: STEPS Survey Nepal 2013. Nepal Health Research Council (NHRC); 2014. http://nhrc.gov. np/wpcontent/uploads/2017/02/noncommunicable-disease-report_2012_2013. pdf. Accessed on May 3 2019.

14. Prevalence, distribution and correlates of tobacco smoking and chewing in Nepal: a secondary data analysis of Nepal Demographic and Health Survey-2006 Sreeramareddy CT, Ramakrishnareddy N, Kumar HH, Sathian B, Arokiasamy JT. Prevalence, distribution and correlates of tobacco smoking and chewing in Nepal: a secondary data analysis of Nepal Demographic and Health Survey-2006. Substance abuse treatment, prevention, and policy. 2011 Dec;6(1):33.

15. Ministry of Health, Nepal; New ERA; and ICF. 2017. Nepal Demographic and Health Survey 2016. Kathmandu: Ministry of Health, Nepal.

16. Tata Institute of Social Sciences (TISS). Mumbai and Ministry of Health and Family Welfare, Government of India. Global Adult Tobacco Survey GATS 2 India 2016-

17.https://mohfw.gov.in/sites/default/ files/GlobaltobacoJune 2018.pdf. Accessed on April 22, 2019.

17. Reddy KS, Gupta PC. Tobacco control in India. New Delhi: Ministry of Health and Family Welfare, Government of India. 2004:43 - 7. https://www.who.int/fctc/reporting/Annex6_Report_on_Tobacco_Control_in_India_2004.pdf. Accessed April 22, 2019.

18. Chang JT, Levy DT, Meza R. Trends and factors related to smokeless tobacco use in the United States. Nicotine Tob Res. 2016 Mar;19(8):1740-8. 18(.

19. Garmienè A, Žemaitienè N, Zaborskis A. Family time, parental behaviour model and the initiation of smoking and alcohol use by ten-year-old children: an epidemiological study in Kaunas, Lithuania. BMC Public Health. 2006 Dec;6(1):287. https://dx.doi.org/10.1186\%2F1471-2458-6-287.

20. US Department of Health and Human Services. National Institute on Alcohol Abuse and Alcoholism. Alcohol and tobacco. Alcohol Alert 71. 2007;71:1-6.

21. Gupta R, Gurm H, Bartholomew JR. Smokeless tobacco and cardiovascular risk. Arch Intern Med. 2004 Sep;27(17):1845-9. 164(.

22. $10.1016 /$ j.ypmed 
Smith PH, Bessette AJ, Weinberger AH, Sheffer CE, McKee SA. Sex/gender differences in smoking cessation: a review. Prev Med. 2016 Nov 1;92:135 - 40.https://doi.org/10.1016/j.ypmed. 2016. 07.013.

23. 10.1093 /ntr/ nts100

Roberts B, Gilmore A, Stickley A, Kizilova K, Prohoda V, Rotman D, Haerpfer C, McKee M. Prevalence and psychosocial determinants of nicotine dependence in nine countries of the former Soviet Union. Nicotine Tob Res. 2012 Apr 22;15(1):271-6. https://doi.org/10.1093 /ntr/ nts100.

24. Heatherton TF, Kozlowski LT. Nicotine addiction and its assessment. Ear Nose Throat J. 1992;69(11):763-7.

25. Moylan S, Jacka FN, Pasco JA, Berk M. Cigarette smoking, nicotine dependence and anxiety disorders: a systematic review of population-based, epidemiological studies. BMC Med. 2012 Dec;10(1):123. https://dx.doi.org/10.1186\%2F1741-7015-10-123.

26. Jones RT, Benowitz NL. Therapeutics for nicotine addiction. https://acnp.org/wpcontent/uploads/2017/11/C107_1533-1544.pdf. Accessed April 22,2019.

27. Schnoll RA, Goren A, Annunziata K, Suaya JA. The prevalence, predictors and associated health outcomes of high nicotine dependence using three measures among US smokers. Addiction. 2013 Nov;108(11):1989-2000. https://doi.org/10.1111/add.12285.

28. Althubaiti A. Information bias in health research: definition, pitfalls, and adjustment methods. J Multidiscip Healthc. 2016;9:211. https://dx.doi.org/10.2147\%2FJMDH.S104807.

29. Grimm P. Social desirability bias. Wiley international encyclopedia of marketing. 2010 Dec 15. https://doi.org/10.1002/9781444316568.wiem 02057.

30. Verification S. Biochemical verification of tobacco use and cessation. Nicotine Tob Res. 2002;4(2):149-59. https://doi.org/10.1080/14622200210123581.

31. Etikan I, Musa SA, Alkassim RS. Comparison of convenience sampling and purposive sampling. American journal of theoretical and applied statistics. 2016 Jan 5;5(1):1-4.

32. 10.1136/tobaccocontrol-2011-050012

Jung-Choi KH, Khang YH, Cho HJ. Hidden female smokers in Asia: a comparison of self-reported with cotinine-verified smoking prevalence rates in representative national data from an Asian population.Tob Control. 2012 Nov 1;21(6):536 - 42. doi:10.1136/tobaccocontrol-2011-050012.

\section{Supplementary Files}

This is a list of supplementary files associated with this preprint. Click to download.

- supplement5.docx 